\title{
The JCMT Transient Survey: An Extraordinary Submillimeter Flare in the T Tauri Binary System JW 566
}

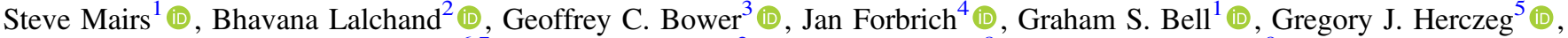 \\ Doug Johnstone $^{6,7}$ (i), Wen-Ping Chen ${ }^{2}$ (i), Jeong-Eun Lee ${ }^{8}$ (i), and Alvaro Hacar ${ }^{9}$ (i) \\ ${ }^{1}$ East Asian Observatory, 660 N. A'ohoku Place, Hilo, HI 96720, USA; s.mairs@eaobservatory.org \\ ${ }^{2}$ Graduate Institute of Astronomy, National Central University, 300 Jhongda Road, Zhongli, Taoyuan 32001, Taiwan \\ ${ }^{3}$ Academia Sinica Institute of Astronomy and Astrophysics, 645 N A'ohoku Place, Hilo, HI 96720, USA \\ ${ }^{4}$ Centre for Astrophysics Research, School of Physics, Astronomy and Mathematics, University of Hertfordshire, College Lane, Hatfield AL10 9AB, UK \\ ${ }^{5}$ Kavli Institute for Astronomy and Astrophysics, Peking University, Yiheyuan Lu 5, Haidian Qu, 100871 Beijing, Peoples Republic of China \\ ${ }^{6}$ NRC Herzberg Astronomy and Astrophysics, 5071 West Saanich Road, Victoria, BC, V9E 2E7, Canada \\ 7 Department of Physics and Astronomy, University of Victoria, Victoria, BC, V8P 5C2, Canada \\ ${ }^{8}$ School of Space Research, Kyung Hee University, 1732, Deogyeong-Daero, Giheung-gu Yongin-shi, Gyunggi-do 17104, Republic of Korea \\ ${ }^{9}$ Leiden Observatory, Leiden University, P.O. Box 9513, 2300-RA Leiden, The Netherlands \\ Received 2018 August 31; revised 2018 November 23; accepted 2018 November 24; published 2019 January 23
}

\begin{abstract}
The binary T Tauri system JW 566 in the Orion Molecular Cloud underwent an energetic, short-lived flare observed at submillimeter wavelengths by the SCUBA-2 instrument on 2016 November 26 (UT). The emission faded by nearly $50 \%$ during the 31 minute integration. The simultaneous source fluxes averaged over the observation are $500 \pm 107 \mathrm{mJy}^{\text {beam }}{ }^{-1}$ at $450 \mu \mathrm{m}$ and $466 \pm 47 \mathrm{mJy} \mathrm{beam}^{-1}$ at $850 \mu \mathrm{m}$. The $850 \mu \mathrm{m}$ flux corresponds to a radio luminosity of $L_{\nu}=8 \times 10^{19} \mathrm{erg} \mathrm{s}^{-1} \mathrm{~Hz}^{-1}$, approximately one order of magnitude brighter (in terms of $\nu L_{\nu}$ ) than that of a flare of the young star GMR-A, detected in Orion in 2003 at $3 \mathrm{~mm}$. The event may be the most luminous known flare associated with a young stellar object and is also the first coronal flare discovered at submillimeter wavelengths. The spectral index between 450 and $850 \mu \mathrm{m}$ of $\alpha=0.11$ is broadly consistent with nonthermal emission. The brightness temperature was in excess of $6 \times 10^{4} \mathrm{~K}$. We interpret this event to be a magnetic reconnection that energized charged particles to emit gyrosynchrotron/synchrotron radiation.
\end{abstract}

Key words: ISM: jets and outflows - stars: formation - stars: variables: general - surveys

\section{Introduction}

Young stellar objects (YSOs) host a range of high-energy phenomena, pointing toward magnetic activity (e.g., Feigelson \& Montmerle 1999; Benz \& Güdel 2010). Of the variability of YSOs across the electromagnetic spectrum, radio and X-ray variability are most closely related to magnetic activity, and indeed YSOs show strong, very short lived flares in these wavelength ranges. X-ray flares of this nature have been comparatively well-studied (e.g., Getman et al. 2008a, 2008b), whereas observations of greater numbers of nonthermal radio flares on timescales of hours or less have only recently become possible with sensitivity improvements, and thus comparably few sources such as these are known at this time. So far, the majority of observations of radio variability have been made on timescales of $\sim$ days to years.

The first example of a YSO radio flare was reported by Bower et al. (2003) toward the weak-line T Tauri star (class III YSO) GMR-A. The flux density at an observing frequency of $86 \mathrm{GHz}$ rose by more than a factor of 5 within a few hours. $\mathrm{X}$-ray monitoring of the source also revealed contemporaneous activity. Follow-up millimeter observations were performed by Furuya et al. (2003), who observed subsequent flare activity for $\sim$ two weeks. Another strong flare at an observing frequency of $90 \mathrm{GHz}$ was reported by Massi et al. (2006) toward the weakline T Tauri star V773 Tau, which also happened to be the first case with evidence for interbinary magnetic interaction. Salter et al. (2008) observed a $3 \mathrm{~mm}$ flare associated with the DQ Tau and showed evidence that these events may be expected for similar binary systems. Even the earliest evolutionary stages of a forming star show activity: Forbrich et al. (2008) reported a strong flare at $22 \mathrm{GHz}$ toward a very deeply embedded protostar in the Orion $\mathrm{BN} / \mathrm{KL}$ region. In all of these cases, the radio emission is interpreted as nonthermal radiation.

Giant flares from young stars are important both for understanding magnetic reconnection as well as for evaluating the impact of high-energy photons on protoplanetary disks and atmospheric escape (e.g., Güdel et al. 2014). The X-ray emission from flares, produced by hotter gas than quiescent $\mathrm{X}$-ray emission, emits energetic photons that penetrate deep into the disk (Glassgold et al. 2004), thereby affecting midplane ionization and chemistry (e.g., Rab et al. 2017; Fraschetti et al. 2018). Variability has been detected in $\mathrm{H}^{13} \mathrm{CO}^{+}$emission and attributed to flares (Cleeves et al. 2017). Flash-heating from $\mathrm{X}$-rays is a possible explanation for producing chondrites (Shu et al. 1997) and abundances of calcium-rich inclusions seen in meteorites (Sossi et al. 2017). Once planets form, the ultraviolet and X-ray emission and cosmic rays produced by magnetic reconnection affects the atmospheric chemistry and enhances their escape (e.g., Lammer et al. 2018).

The correlation between X-ray and radio variability in YSOs has remained unclear, however, even though there is undoubtedly an underlying connection (e.g., Güdel \& Benz 1993). Recently, Forbrich et al. (2016, 2017) analyzed the incidence of order-of-magnitude flaring variability on short timescales in the Orion Nebula Cluster, using simultaneous radio $(4.7$ and $7.3 \mathrm{GHz}$ ) and $\mathrm{X}$-ray observations. They found that there is a close connection only for a subset of this extreme radio and X-ray variability. 
In this paper, we present the detection of a submillimeterwavelength outburst from the YSO JW 566 in Orion, discovered in the James Clerk Maxwell telescope (JCMT)Transient submillimeter monitoring program of nearby starforming regions (Herczeg et al. 2017). This flare falls into the same category as the short timescale, likely nonthermal radio flares described above. The short timescale for the decay of the flare rules out reprocessed accretion luminosity through thermal dust emission in the envelope (Johnstone et al. 2013) as the source of variability, which until this paper had been the type of variability uncovered within our survey (Mairs et al. 2017a; Yoo et al. 2017; Johnstone et al. 2018), even though this observation by itself does not necessarily imply entirely distinct emission mechanisms. This is the first such flare discovered at submillimeter wavelengths. In Section 2, we present details of our observations and data reduction methods. In Section 3, we introduce the methods used to detect the flare. In Section 4, we display the 450 and $850 \mu \mathrm{m}$ images and construct a short timescale light curve of the flare. In Section 5, we discuss the results and compare the data presented in this paper to previous observations of JW 566 at other wavelengths. Finally, in Section 6, we summarize our findings.

\section{Observations and Data Reduction}

\subsection{SCUBA-2 Observations}

The JCMT Transient Survey (project code: M16AL001; Herczeg et al. 2017) has been monitoring submillimeter emission from the Orion Molecular Cloud (OMC) 2/3 region, centered at (R.A., decl. $)=(05: 35: 33,-5: 00: 32$, J2000), and seven other nearby star-forming regions with a roughly monthly cadence since 2015 December. Each region is observed with SCUBA-2 (Holland et al. 2013) at 450 and $850 \mu \mathrm{m}$ simultaneously, with beam sizes of 9". 8 and 14 ." 6 , respectively (Dempsey et al. 2013). The images are constructed using $2^{\prime \prime}$ pixels at $450 \mu \mathrm{m}$ and $3^{\prime \prime}$ pixels at $850 \mu \mathrm{m}$. The SCUBA-2 observing mode Pong1800 (Kackley et al. 2010) yields maps with a smooth sensitivity over a circular region with $30^{\prime}$ diameter. Our integration times are set to ensure a consistent background noise level of $\sim 10 \mathrm{mJy}^{\text {beam }}{ }^{-1}$ at $850 \mu \mathrm{m}$ from epoch to epoch (Mairs et al. 2017b). At $450 \mu \mathrm{m}$, the submillimeter emission from the atmosphere has a much more significant effect on the data, producing a range of noise levels that span more than an order of magnitude.

Table 1 provides a log for our observations of OMC 2/3. The flare is detected in our observations of 2016 November 26, beginning at $\mathrm{MJD}=57718.453$ and ending at $\mathrm{MJD}=57718.474$. We also include in our analysis of JW 566 engineering/ commissioning observations (project code M16BEC30) obtained on 2016 November 20 (UT), 6 days before the flare, which were obtained to assess the health of SCUBA-2 after maintenance. This engineering observation is used only to determine the detectability of JW 566, and is excluded from our coadded images and global analysis.

\subsection{Data Reduction and Flux Uncertainties}

The data reduction was carried out using the iterative mapmaking software, MAKEMAP (see Chapin et al. 2013 for details), which is part of STARLINK's (Currie et al. 2014) Submillimetre User Reduction Facility (SMURF) package (Jenness et al. 2013). The specific data reduction and image calibration techniques used by the JCMT Transient Survey are
Table 1

A Summary of the OMC $2 / 3$ Observations $^{\mathrm{a}}$ Performed by the JCMT Transient Survey to Date

\begin{tabular}{|c|c|c|c|c|c|}
\hline $\begin{array}{l}\text { UT Date } \\
\text { (YYYY- } \\
\text { MM-DD) }\end{array}$ & $\mathrm{Scan}^{\mathrm{b}}$ & $\tau_{225}{ }^{\mathrm{c}}$ & Airmass & $\begin{array}{c}850 \mu \mathrm{m} \\
\sigma_{\mathrm{rms}} \\
\left(\mathrm{mJy}^{-1}\right) \\
\left.\text { beam }^{-1}\right)\end{array}$ & $\begin{array}{c}450 \mu \mathrm{m} \\
\sigma_{\mathrm{rms}}^{\mathrm{d}} \\
(\mathrm{mJy} \\
\left.\text { beam }^{-1}\right)\end{array}$ \\
\hline 2015 Dec 26 & 36 & 0.11 & 1.13 & 9.9 & 411.2 \\
\hline 2016 Jan 16 & 19 & 0.06 & 1.21 & 7.7 & 81.3 \\
\hline 2016 Feb 06 & 12 & 0.04 & 1.36 & 9.7 & 73.4 \\
\hline 2016 Feb 29 & 11 & 0.04 & 1.16 & 9.4 & 67.4 \\
\hline 2016 Mar 25 & 15 & 0.06 & 1.27 & 8.7 & 101.8 \\
\hline 2016 Apr 22 & 11 & 0.05 & 1.72 & 9.1 & 126.4 \\
\hline 2016 Aug 26 & 20 & 0.11 & 1.26 & 12.1 & 398.6 \\
\hline 2016 Nov $20^{e}$ & 20 & 0.084 & 1.31 & 7.9 & 174.8 \\
\hline 2016 Nov 26 & 52 & 0.06 & 1.11 & 7.7 & 85.2 \\
\hline 2017 Feb 06 & 21 & 0.12 & 1.15 & 9.8 & 392.5 \\
\hline 2017 Mar 18 & 12 & 0.10 & 1.10 & 9.1 & 255.6 \\
\hline 2017 Apr 21 & 22 & 0.09 & 1.41 & 10.4 & 382.8 \\
\hline 2017 Jul 08 & 73 & 0.05 & 1.24 & 9.4 & 72.1 \\
\hline 2017 Aug 12 & 56 & 0.07 & 1.60 & 8.5 & 187.1 \\
\hline 2017 Sep 12 & 54 & 0.10 & 1.32 & 9.0 & 280.3 \\
\hline 2017 Oct 21 & 47 & 0.09 & 1.11 & 8.0 & 164.8 \\
\hline 2017 Nov 18 & 46 & 0.06 & 1.10 & 9.5 & 77.6 \\
\hline 2017 Dec 24 & 46 & 0.07 & 1.36 & 8.5 & 148.5 \\
\hline 2018 Feb 08 & 18 & 0.09 & 1.20 & 8.0 & 184.6 \\
\hline 2018 Mar 08 & 15 & 0.11 & 1.64 & 10.4 & 780.1 \\
\hline
\end{tabular}

Notes. Bold text highlights the date of the flare event (see Section 5).

${ }^{a}$ The target identifier in the JCMT archive is OMC2-3.

$\mathrm{b}$ The observation number.

${ }^{\mathrm{c}} \tau_{225}$ is the zenith opacity of the atmosphere at $225 \mathrm{GHz}$.

d Noise measurements are based on the pixel variances in a central $900^{\prime \prime}$ radius of the Gaussian smoothed map.

e This data was taken as part of an engineering and commissioning project (project ID: M16BEC30) and not as part of the JCMT Transient Survey.

described in detail by Mairs et al. (2017b; reduction $R 3$ with no relative flux calibration applied ${ }^{10}$ ). Briefly, to perform a Pong1800 observation, the telescope continually scans across the sky, observing each location at a variety of position angles. This technique allows for the modeling and subtraction of the large-scale, bright, variable atmosphere at submillimeter wavelengths. The continual scanning across the sky also provides a time-series that is exploited in this paper to measure light curves during our observation.

To optimize the extraction of compact sources, we applied a stringent spatial filter during the data reduction to suppress the signal on scales $>200^{\prime \prime}$. A mask was used to define significant astronomical emission in the image, which provided additional constraints to MAKEMAP and aided in the background subtraction (see Chapin et al. 2013; Mairs et al. 2015). The OMC $2 / 3$ region is particularly difficult to mask due to the large amount of extended emission, though all persistent point sources are well accounted for in the regular analysis pipeline. The source with a strong flare, JW 566, resides in a region of negative bowling, a section of the image with artificially low values just outside the boundaries of bright, extended emission, due to the application of the stringent spatial filter. The external mask was therefore adjusted to include the bright event

\footnotetext{
${ }^{10}$ To suppress the false detections of noise spikes or artificial structure, the images are smoothed with Gaussian kernels with FWHM values of $4^{\prime \prime}$ and $6^{\prime \prime}$ at $450 \mu \mathrm{m}$ and $850 \mu \mathrm{m}$, respectively (two pixels in each case).
} 
associated with JW 566 and the data reduction was rerun for all epochs to ensure the flux of the source was well recovered.

In addition, all Transient Survey epochs, excluding 2016 November 26 (the date of the flare event), were then coadded and subtracted from the 2016 November 26 data to produce residual flux maps at 450 and $850 \mu \mathrm{m}$. The fluxes of JW 566 quoted in Section 4 were measured in the residual maps to suppress any background structure present in the image. At $850 \mu \mathrm{m}$, the background level of the coadd at the location of JW 566 is $2 \mathrm{mJy}$ beam $^{-1}$, which is insignificant relative to the peak flux measurement of the source. We adopt the JCMT standard flux uncertainty value of $10 \%$ for $850 \mu \mathrm{m}$ observations (Dempsey et al. 2013; S. Mairs et al. 2018, in preparation). At $450 \mu \mathrm{m}$, however, JW 566 is located within a negative bowl in the coadded image, with a background level of $-100 \mathrm{mJy}^{-1}$ beam $^{-1}$. The standard deviation of the mean negative bowl depth within the region of JW 566 for observations with similar background noise $\left(450 \mu \mathrm{m} \sigma_{\text {rms }}<\right.$ $120 \mathrm{mJy} \mathrm{beam}^{-1}$ ) is $77 \mathrm{mJy}^{\text {beam }}{ }^{-1}$ (see Table 1 ). We therefore combine the JCMT standard flux uncertainty value at $450 \mu \mathrm{m}$ of $15 \%$ (Dempsey et al. 2013; S. Mairs et al. 2018, in preparation) with the uncertainty in the negative bowl depth for an uncertainty of $21 \%$ for JW 566 .

The fluxes described in this paper ignore any emission from ${ }^{12} \mathrm{CO} \mathrm{J}=3-2$, which is located within the $850 \mu \mathrm{m}$ filter of SCUBA-2 (for a discussion on the effects of CO contamination, see Drabek et al. 2012; Coudé et al. 2016; Parsons et al. 2018). In the JCMT Gould Belt Survey image of ${ }^{12} \mathrm{CO}$ emission obtained with the Heterodyne Array Receiver Programme (HARP) (Buckle et al. 2009), weak CO emission is dispersed across the location of JW 566 with no significant compact structure, and has a negligible effect on the continuum emission described in this paper.

\subsection{Subdividing the Raw Data}

Raw SCUBA-2 data are comprised of the power received at the focal plane over time, with separate integrations read out and saved in $\sim 36 \mathrm{~s}$ intervals, some of which include observations of JW 566. In Section 4.3, we subdivide this data stream into nine shorter integrations based on when the telescope passed over JW 566. These subdivisions are reconstructed into individual images using the same external mask and data reduction parameters as for the full integration.

\section{Searching for Variability of Faint Sources}

At the time of writing, the JCMT Transient Survey has obtained nearly $3 \mathrm{yr}$ of data across eight star-forming regions. Johnstone et al. (2018) analyzed source variability in all $850 \mu \mathrm{m}$ images taken throughout the first 18 months of the survey (2015 December through 2017 May). The 1643 sources in that analysis were selected by identifying compact emission peaks with a brightness five times higher than the noise in the coadded maps of each region in our survey. Since that time, these same sources have been tracked with an automated pipeline that measures the flux soon after the data are obtained and compares it with past observations. The automated version of the Johnstone et al. (2018) analysis, however, relies on the initial detection of a source in the coadded image of a given target field. Therefore, the pipeline is only sensitive to short timescale burst events that are strong enough to be detected at a level of $5 \sigma_{\mathrm{rms}}$ in the coadd. We are now in the process of further evaluating transient variability by searching for any sources that might have appeared in only a single image (B. Lalchand et al. 2018, in preparation). Following the methods of Johnstone et al. (2018), we use the JSA_CATALOGUE program (found in STARLINK's PICARD package Gibb et al. 2013) to optimize and run the FELLWALKER (Berry 2015) source detection algorithm to identify compact, peaked, continuum emission structures. While Johnstone et al. (2018) used this strategy to produce a source catalog from the coadded image of a field, we repeat their procedure for each individual epoch in the OMC 2/3 region. In this way, we compare the catalogs generated for each epoch with the catalog generated for the coadd and identify sources that appeared in individual observations, but not in the averaged image. We refer to these sources as candidate transients.

Figure 1 shows the $850 \mu \mathrm{m}$ source peak (the maximum pixel value in each source footprint) of all the sources detected in the OMC 2/3 coadd (gray) versus the effective diameter. The effective diameter of a source is calculated by measuring the total area in the identified clump footprint and assuming a projected circular symmetry. Since this method takes into account the full clump size (and not the brightness profile), unresolved sources will appear larger than the beam FWHM, while false detections due to residual correlated noise in the final image will appear smaller than the beam FWHM. The circular symmetry assumption holds well for compact (approximately beam-sized) objects. Also included (green) are the sources detected only in individual epochs. Several faint, spurious sources that do not appear at the level of $5 \sigma_{\text {rms }}$ in the coadd and are smaller than the beam are detected in single epochs. A full, multiregion analysis of candidate transients like these will be presented by B. Lalchand et al. (2018, in preparation).

One unresolved ${ }^{11}$ source, however, is a clear outlier. This source is detected at (R.A., decl.) $=(5: 35: 17.94,-5: 16: 11)$ on 2016 November 26 (UT), with an initial $850 \mu \mathrm{m}$ source peak of $384 \mathrm{mJy}^{\text {beam }}{ }^{-1}$ in an observation that had a background noise level of $\sigma_{\mathrm{rms}}=9.74 \mathrm{mJy}$ beam $^{-1}(\mathrm{~S} / \mathrm{N}=39)$. This flux, however, is underestimated as it was measured before the image was rereduced with an appropriate mask (see Section 2.2). The 2016 November 26 epoch is the only image with a detection of this source. The source was not identified in our initial measurements of variability from the first 11 epochs of the survey (Johnstone et al. 2018) because negative bowling in the region of JW 566 led to an average source brightness of $\sim 0.8$ mJy beam $^{-1}$ at the peak pixel location.

The position of this candidate transient peak is within $2 . " 4$ of the position of JW 566 (Jones \& Walker 1988), a K7+M1.5 T Tauri binary system with a projected separation of $0 . " 86$ (Daemgen et al. 2012). It has been classified as a "Disk" by Megeath et al. (2012) based on its midinfrared colors.

\section{A Submillimeter Flare of JW 566}

\subsection{Detecting the Flare at $850 \mu \mathrm{m}$}

Out of 20 epochs of SCUBA-2 imaging, only one (2016 November 26) shows bright, unresolved $850 \mu \mathrm{m}$ emission at the location of JW 566 (see Table 1 and Figure 2). Figure 2

\footnotetext{
11 A Gaussian fit at the position of the significant candidate transient source in the smoothed, $850 \mu \mathrm{m}$ image results in a full width at half maximum value of 15 " 5 averaged over the vertical and horizontal directions. The effective beam size after smoothing is $15^{\prime \prime} .8$.
} 


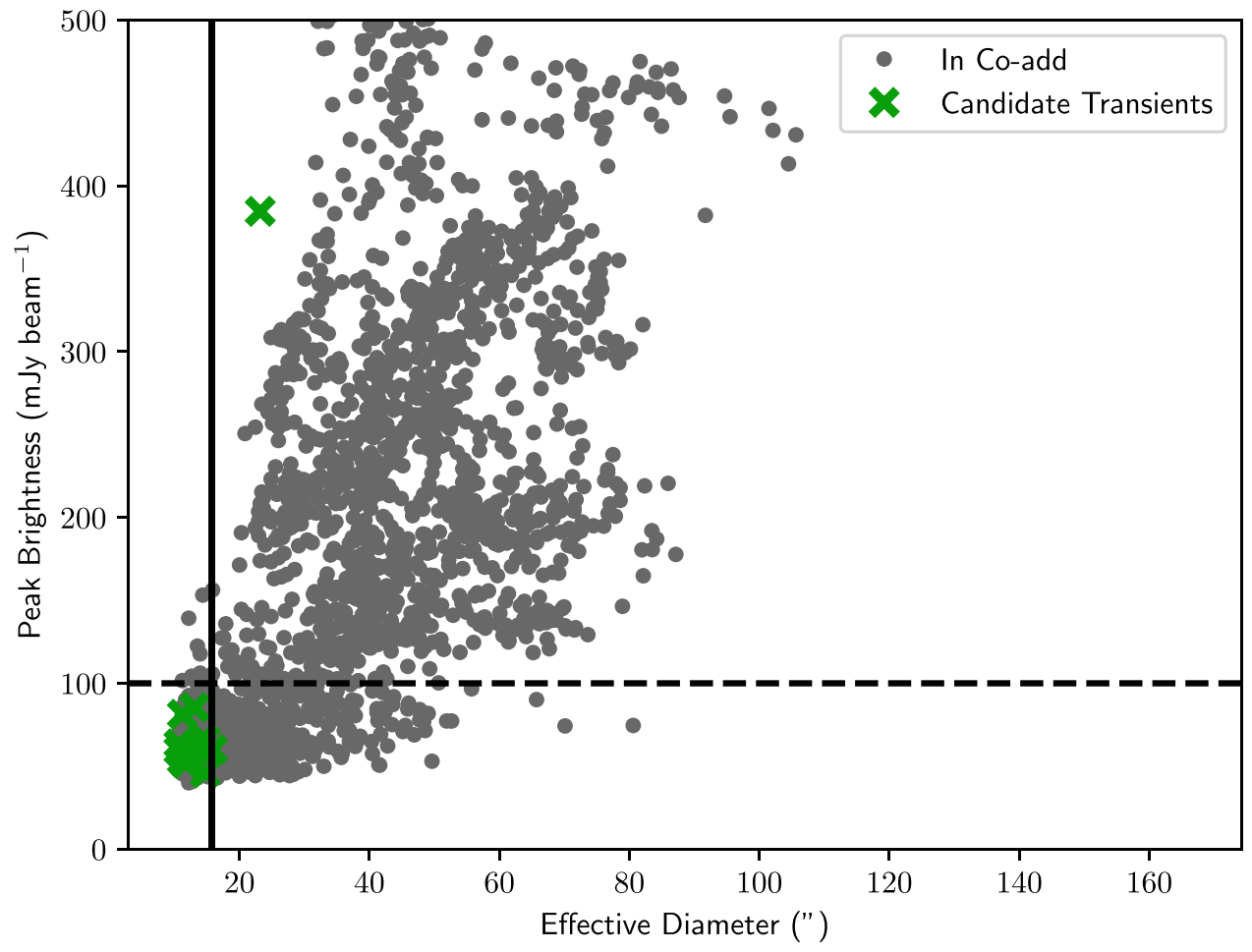

Figure 1. The $850 \mu \mathrm{m}$ peak brightness vs. the effective diameter (assuming a circular projection) of all sources identified in all 19 OMC $2 / 3$ epochs. Gray circles indicate sources identified in the coadd that are being analyzed by the current pipeline. Green X's indicate candidate transient sources that do not appear in the coadd (at the level of $5 \sigma_{\mathrm{rms}}$ ) but do appear in at least one epoch. A horizontal (dashed) line has been drawn at $100 \mathrm{mJy} / \mathrm{beam} \sim 10 \sigma_{\mathrm{rms}}$. The vertical (solid) line represents the effective $850 \mu \mathrm{m}$ beam FWHM after Gaussian smoothing.

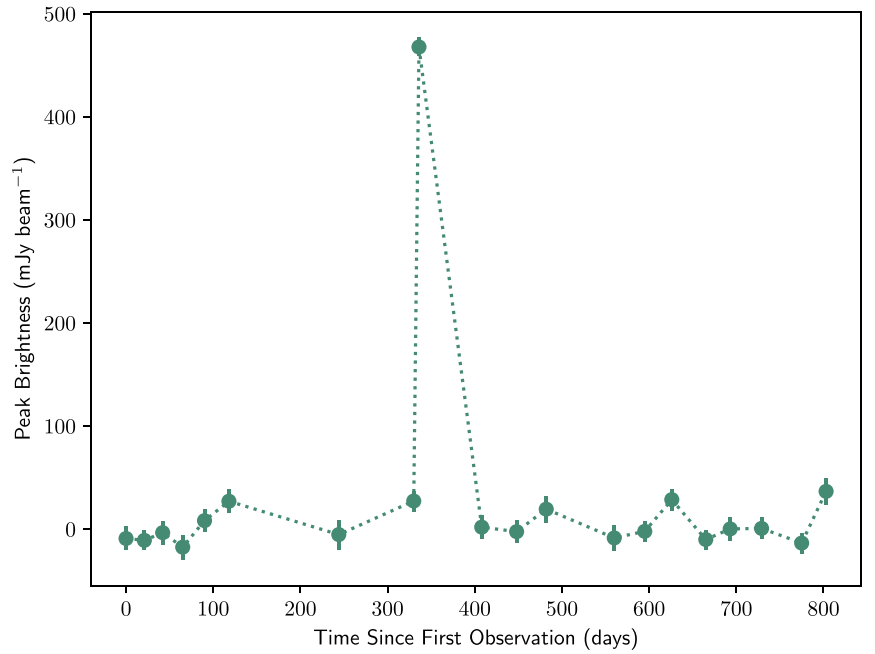

Figure 2. The $850 \mu \mathrm{m}$ light curve of JW 566 over all observed epochs (see Table 1).

presents a light curve derived from extracting the value of the pixel at the peak location of JW 566. ${ }^{12}$ The uncertainties are calculated by measuring the average pixel variance ${ }^{13}$ in a $20 \times 20$ pixel box centered on the source. Figure 3 shows that emission is not detected at this position in the previous image obtained six days earlier, or in the subsequent image obtained three months later. After coadding all 18 Transient Survey

\footnotetext{
12 The small fluctuations are due to a slight amount of faint, extended emission in this region, which is better recovered in some epochs, but it is not associated with JW 566.

13 Each SCUBA-2 map has an associated "variance map" that records the variance of the bolometer signals contributing to each pixel.
}

epochs without a detection, the source is still not detected, with a noise of $\sigma_{\mathrm{rms}}=3 \mathrm{mJy}$ beam $^{-1}$ in the coadded map (indicating an upper limit on the flux of $\sim 3 \sigma_{\text {rms }}=9 \mathrm{mJy} \mathrm{beam}^{-1}$ ). The source is also not detected in the coadd of the SCUBA-2 images obtained by the JCMT Gould Belt Survey (WardThompson et al. 2007) in 2011, with a sensitivity of $\sim 4$ mJy beam $^{-1}$ (Data Release 3; Kirk et al. 2018).

The bright peak associated with JW 566 is detected in the map obtained during a 31 minute observation. The excess emission is consistent with an unresolved object at the (nonsmoothed) 14!" 6 resolution of the JCMT. The source is best detected in a residual map of the coadd (Figure 3(d)) subtracted from the flare epoch (Figure 3(b)) (after the images were rereduced with the new mask; see Section 2.2). The average brightness of the source during our observation is $466 \pm 47 \mathrm{mJy}^{-1}$ beam $^{-1}(\mathrm{~S} / \mathrm{N}=48)$, as measured by fitting a Gaussian profile to the source in the residual map.

\subsection{Detecting the Flare at $450 \mu \mathrm{m}$}

The brightness peak of JW 566 is also detected in the simultaneous $450 \mu \mathrm{m}$ images obtained with SCUBA-2. The precipitable water vapor was low on the night of the flare, leading to a noise level of $\sigma_{\mathrm{rms}}=85 \mathrm{mJy}$ beam $^{-1}$. Figure 4 presents the coadded data in image (a), excluding the 2016 November 26 epoch, the 2016 November 26 (flare) epoch in image (b), and a subtraction of image (a) from image (b) in image (c).

As in the case of the $850 \mu \mathrm{m}$ observations, there is no indication of significant emission correlated with the position of JW 566 in any other SCUBA-2 image. A two-dimensional Gaussian profile fit to the residual $450 \mu \mathrm{m}$ image yields a source peak of $500 \pm 107 \mathrm{mJy}$ beam $^{-1}$ (the detection has an 


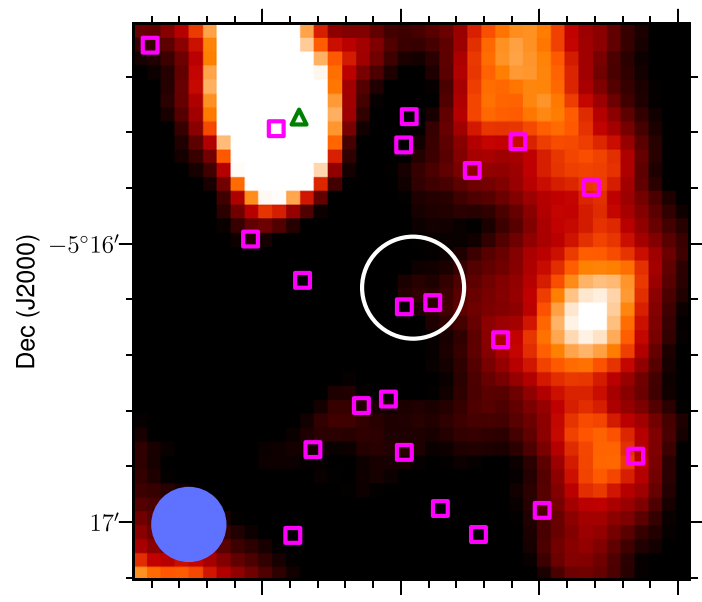

(a) $850 \mu \mathrm{m}$ 2016-11-20 (UT).

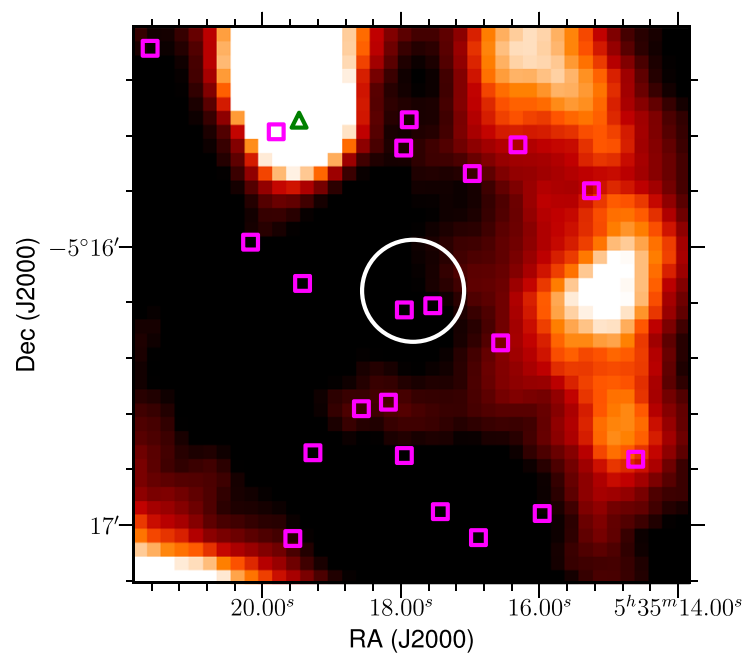

(c) $850 \mu \mathrm{m} \mathrm{2017-02-06} \mathrm{(UT).}$

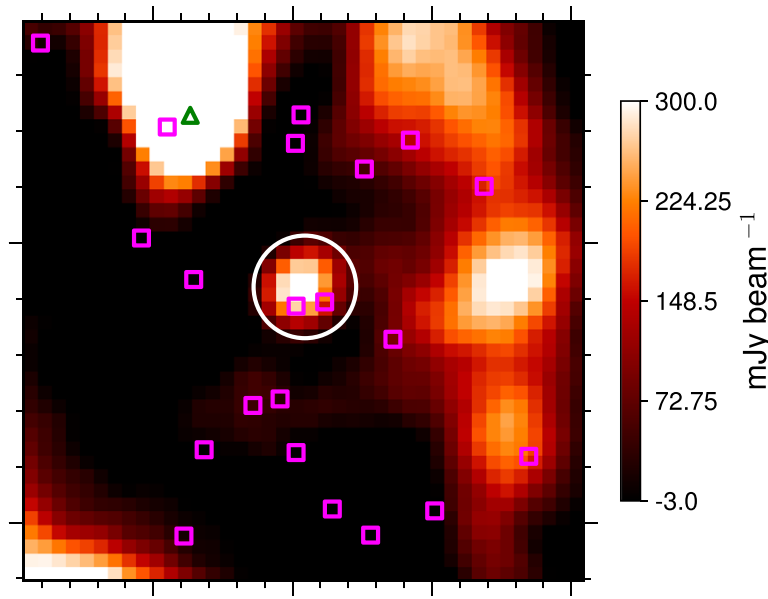

(b) $850 \mu \mathrm{m}$ 2016-11-26 (UT).

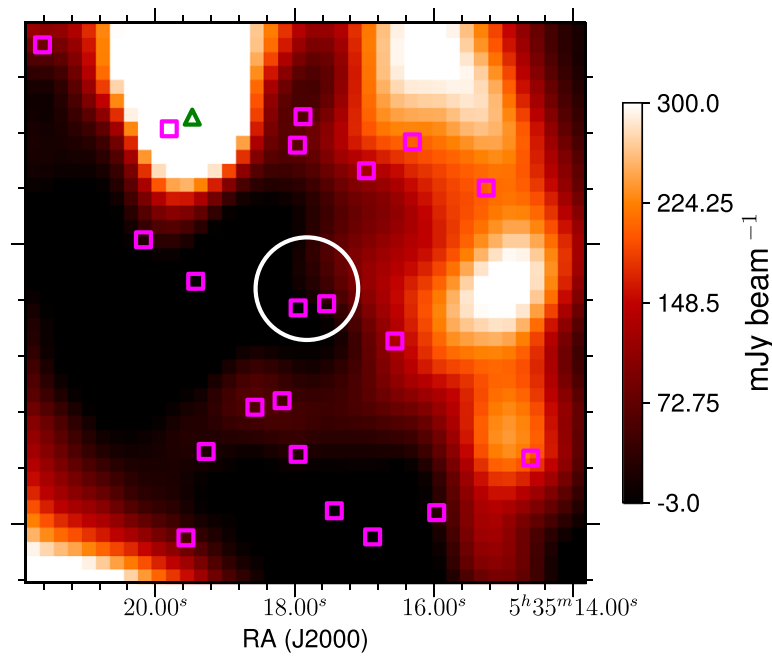

(d) The co-add of all $850 \mu \mathrm{m}$ epochs not including 2016-11-26

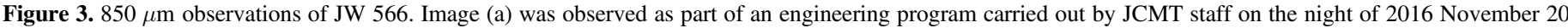

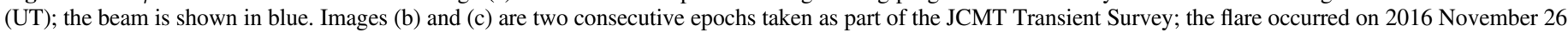

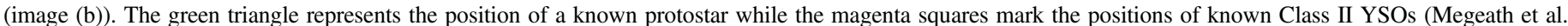
2012). The white circle shows the location of JW 566. Image (d) is a coadd of all $850 \mu$ m epochs not including 2016 November 26.

$\mathrm{S} / \mathrm{N}=6$; see Section 2.2 for more information about the uncertainty).

\subsection{Minute to Minute Variability at $850 \mu \mathrm{m}$}

Since JW 566 is not detected in the engineering data, 6 days prior to the flare, the source must vary on timescales shorter than one week. In this section, we analyze the light curve of the bright emission peak in nine separate intervals within the 31.12 minute integration of 2016 November 26. In each interval, the source is detected with an $\mathrm{S} / \mathrm{N}$ between 5 and 25 and is fit with a two-dimensional Gaussian profile to measure the source peak.

Figure 5 shows a dramatic decay in brightness during the 31 minute integration. The emission from JW 566 appears to already be in the dimming phase of the outburst, with an initial peak of $773 \mathrm{mJy} \mathrm{beam}^{-1}$ that drops to $400 \mathrm{mJy} \mathrm{beam}^{-1}$ by the end of the observation. The uncertainties for each measurement are calculated by measuring the square root average pixel variance in a $20 \times 20$ pixel box centered on the source.
To confirm that this brightness decrease is significant, we also analyze the light curves of five nonvarying unresolved sources with fluxes sampling the range of JW 566 in this epoch. Subdividing the raw time stream was performed in the same way individually for each source as it was for JW 566. Data points with abnormally high variances due to their proximity to the edge of the map or the uncertainty in surrounding, largescale structure have been discarded. All of these sources are consistent with a constant flux during the 31 minute observation. The average standard deviation of the nonvarying sources is $9.7 \%$. The light curve of JW 566 is monotonically decreasing and has a standard deviation of $23.2 \%$, more than twice the average value of the nonvarying sources. At $450 \mu \mathrm{m}$, the noise is too high to perform a similar analysis.

\section{Discussion}

Young stars are known to undergo large, short-lived (timescales of hours to days) outbursts detectable at millimeter 


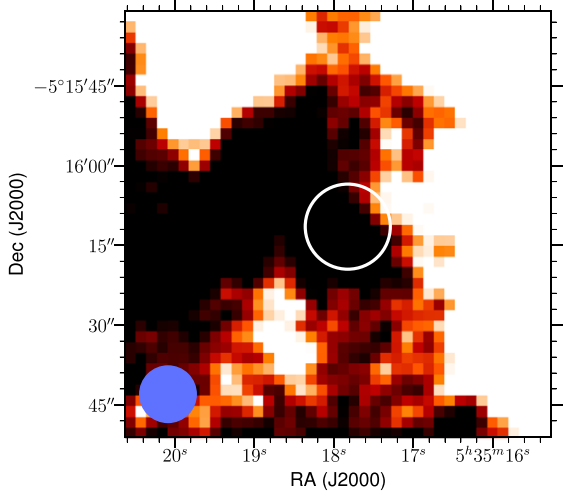

(a) Co-added data (not including observation on 2016-11-26).

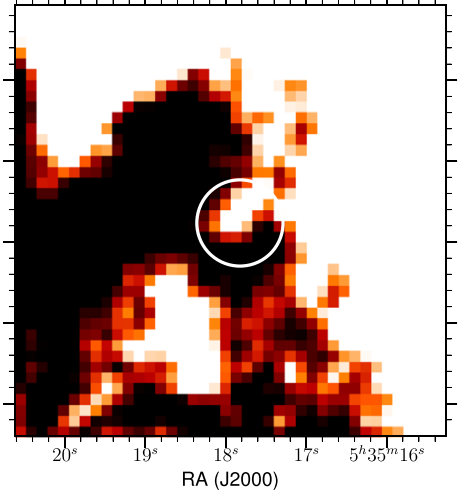

(b) 2016-11-26 (UT).

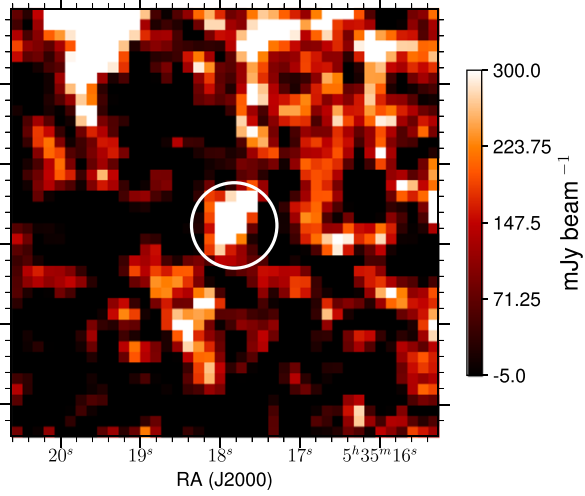

(c) Observation on 2016-11-26 minus the co-add.

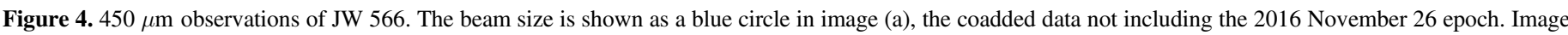

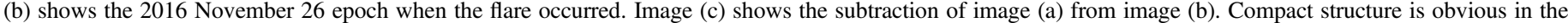
residual.

and centimeter wavelengths (Bower et al. 2003; Furuya et al. 2003; Massi et al. 2006; Salter et al. 2010; Forbrich et al. 2017). Flare studies tend to focus on these frequencies, leaving the submillimeter frequency space of JCMT largely unexplored.

At a distance of $389 \mathrm{pc}^{14}$ the measured $850 \mu \mathrm{m}$ flux $\left(466 \mathrm{mJy}\right.$ beam $\left.^{-1}\right)$ corresponds to a radio luminosity of $L_{\nu}=$ $8 \times 10^{19} \mathrm{erg} \mathrm{s}^{-1} \mathrm{~Hz}^{-1}$. A natural comparison point for this result is with the 2003 outburst event of the T Tauri star GMRA in the Orion Nebula (Bower et al. 2003; see also Furuya et al. 2003). GMR-A had a radio luminosity of $L_{\nu}=3 \times$ $10^{19} \mathrm{erg} \mathrm{s}^{-1} \mathrm{~Hz}^{-1}$ at $86 \mathrm{GHz}$ (assuming the same distance of $389 \mathrm{pc}$ ), which makes the JW 566 flare an order of magnitude brighter in terms of $\nu L_{\nu}$. Salter et al. (2008) and Massi et al. (2006) observed flares associated with the DQ Tau binary system at $115 \mathrm{GHz}$ and the V773 Tau quadruplet at $90 \mathrm{GHz}$, respectively, with radio luminosities of $\sim 6 \times$ $10^{19} \mathrm{erg} \mathrm{s}^{-1} \mathrm{~Hz}^{-1}$. If the flare associated with JW 566 follows the X-ray/radio luminosity correlation (see, for example, Güdel 2002), then it is 10 orders of magnitude brighter than a typical solar flare. It is plausible that this is the most luminous flare ever recorded in a young star. In the future, coordinated observations are required at $450,850 \mu \mathrm{m}$, and other wavelengths to reveal the relationship between the fluxes at different energy regimes.

The OMC $2 / 3$ field has been observed with SCUBA-2 for $10 \mathrm{hr}$ since 2015 December 26, and this is the first significant flare event of its kind discovered in those data. In total, there are $\sim 600$ known (Spitzer identified; Megeath et al. 2012) Class II (disk) objects present in the field of view. Therefore, the current detection rate of flare events of this magnitude is $1 /(600$ stars $\times 10 \mathrm{hr}) \approx 1-2 \mathrm{yr}^{-1} \mathrm{star}^{-1}$. It is likely that there is a luminosity function for submillimeter flares that scales as a power law, $N \propto L^{\beta}$, where $\beta<0$. More and deeper observations of this field, including seven additional hours from our Transient survey by 2020 February, will allow us to measure the flare rate over a wide range of luminosities. Additionally,

\footnotetext{
${ }^{14}$ We adopt a distance of 389 pc to JW 566 due to its proximity with the Orion Nebula Cluster. This is based on the analysis of Kounkel et al. (2018), who used Very Long Baseline Array data (Kounkel et al. 2017) and GAIA DR2 astrometry (Gaia Collaboration et al. 2018).
}

we will be able to perform a more complete search by detecting fainter, longer timescale events by coadding subsets of the data.

The detection of a coronal flare at submillimeter wavelengths adds another source of uncertainty in the measurement of disk masses (e.g., Pascucci et al. 2016). While submillimeter emission from most sources is produced by the thermal dust continuum emission within the protoplanetary disks, any unexpected emission from sources thought to be diskless should be tested to evaluate whether flaring may explain the emission. Indeed, unresolved $1.3 \mathrm{~mm}$ continuum emission from Prox Cen was initially interpreted as an indication of a candidate disk (Anglada et al. 2017) but later traced to a stellar flare (MacGregor et al. 2018).

\subsection{Previous Observations of JW 566}

The JW 566 binary system ( 0 "! 86 projected separation) has a disk around at least one of the components (Megeath et al. 2012). Daemgen et al. (2012) detected accretion around the K7 primary star but not the M1.5 secondary star. High-resolution optical spectra of JW 566 are not available, so it is unknown whether one or both stars are spectroscopic binaries. Interactions between the magnetospheres of close binaries are thought to excite coronal flares in DQ Tau (Salter et al. 2010) and perhaps other young stars.

Previous X-ray and radio observations demonstrate coronal flares from JW 566, as expected for young low-mass stars. Kounkel et al. (2014) classify the source as variable at both 4.5 and $7.5 \mathrm{GHz}$. In addition, JW 566 is a known X-ray source (Gagne et al. 1995; Garmire et al. 2000; Feigelson et al. 2002; Getman et al. 2005), with variability on timescales of hours. The JW 566 binary is one of the most luminous X-ray sources, with $L_{\mathrm{X}}=10^{31} \mathrm{erg} \mathrm{s}^{-1}$, for its mass range in the COUP X-ray monitoring survey of the Orion Nebula (Getman et al. 2005). The extreme brightness is caused by a combination of saturated $\mathrm{X}$-ray emission, with $\log L_{\mathrm{X}} / L_{\mathrm{bol}}=-3$, and large radii as measured by Daemgen et al. (2012). The X-ray emission is harder than average but not extreme among the COUP sample.

The source is detected in a $3 \mathrm{~mm}$ continuum image obtained by ALMA on 2015 December 26 (Hacar et al. 2018; see Figure 6). The $3 \mathrm{~mm}$ flux is measured to be $0.6 \mathrm{mJy}$, yielding 

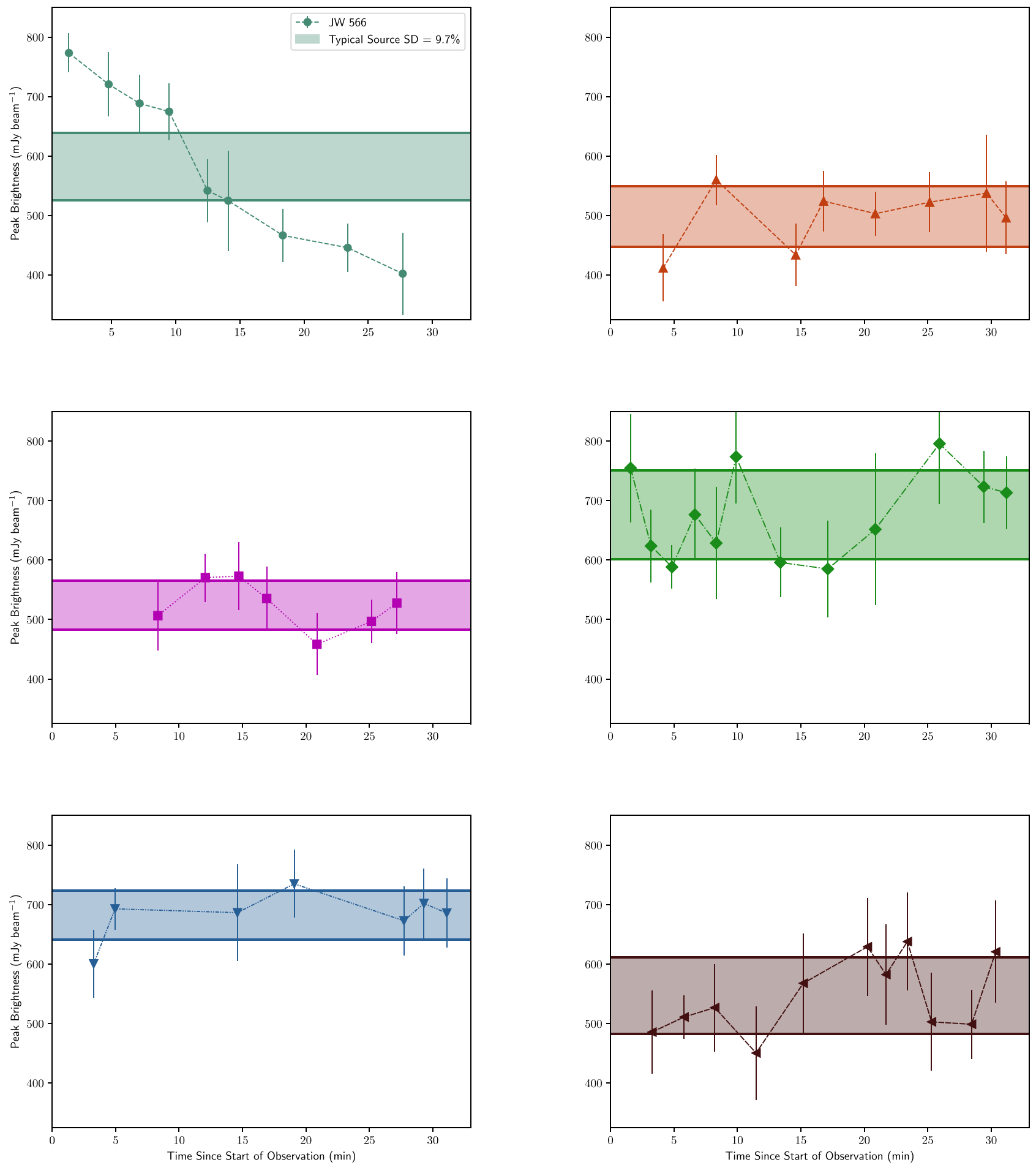

Figure 5. The $850 \mu \mathrm{m}$ peak brightness of JW 566 (top left) along with five typical sources in the brightness range displayed by JW 566 throughout the 31 minute integration. The sources were selected from different locations around the OMC $2 / 3$ field. The light-curve standard deviations are shown by the shaded regions in each plot. The average standard deviation of the nonvarying sources (9.7\%) is overlaid on JW 566's light curve.

an $\mathrm{S} / \mathrm{N}$ of 5.7. This presumably quiescent flux is a factor of $8 \times 10^{2}$ fainter than the $850 \mu$ m continuum measurement of the flare, assuming a spectral index of 1 . If the $3 \mathrm{~mm}$ flux is produced by the disk, a spectral index of $\sim 2.3$ (e.g., Ricci et al. 2010 ) would lead to an $850 \mu \mathrm{m}$ flux of $\sim 10 \mathrm{mJy}$ beam $^{-1}$, very close to our current detection limit in the coadded image and within the flux range expected for disks in nearby star-forming regions (e.g., Ansdell et al. 2016; Pascucci et al. 2016).
Significant variability has not been detected at wavelengths other than millimeters and the X-rays, including in the optical, $J H K_{S}$ (Ali \& Depoy 1995; Tsujimoto et al. 2003; Skrutskie et al. 2006), at 3.6 and $4.5 \mu \mathrm{m}$ (MoralesCalderón et al. 2011), or in the far-IR (Billot et al. 2012). Unfortunately, we do not know of other available data at optical, infrared, or radio wavelengths at the time of this observation. 


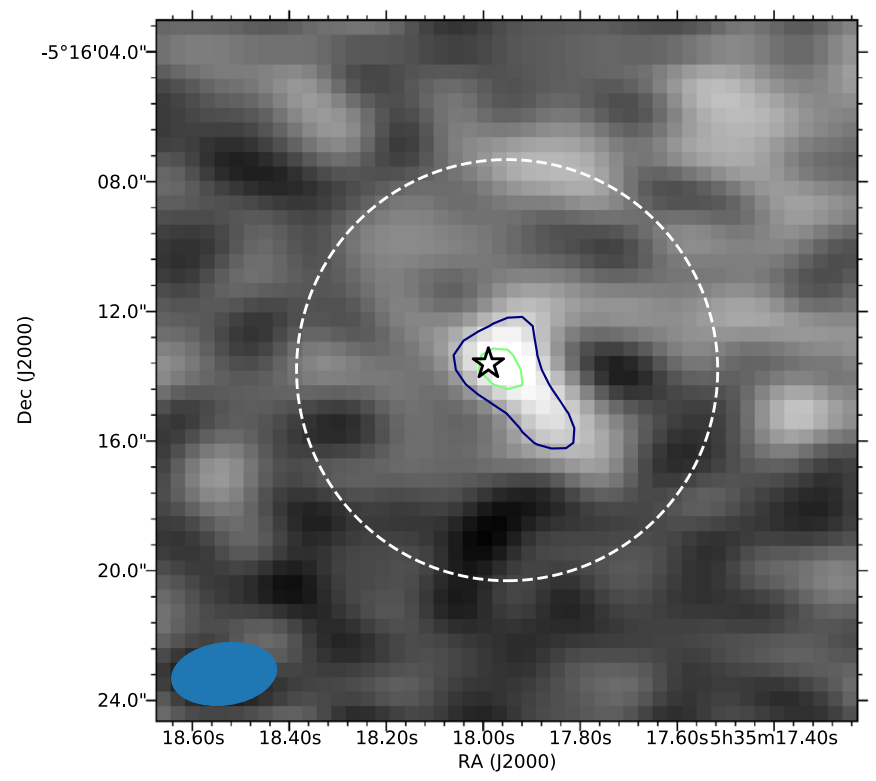

Figure 6. $3 \mathrm{~mm}$ continuum ALMA detection of JW 566 (star symbol). Contours are drawn at $3 \sigma$ and $5 \sigma$. The dashed circle indicates the size of the SCUBA-2 $850 \mu \mathrm{m}$ beam FWHM. The beam is displayed in the lower left.

\subsection{The Nature of JW 566's Flare}

Based on the measured source fluxes at $450 \mu \mathrm{m}(666 \mathrm{GHz}$, $\left.f_{666}=500 \pm 107 \mathrm{mJy} \mathrm{beam}^{-1}\right)$ and $850 \mu \mathrm{m}\left(352 \mathrm{GHz}, f_{352}=\right.$ $466 \pm 46.6 \mathrm{mJy} \mathrm{beam}^{-1}$ ), we calculate a spectral index

$$
\alpha=\frac{\left(\log \left(f_{666}\right) \pm \frac{\Delta f_{666}}{f_{666} \ln (10)}\right)-\left(\log \left(f_{352}\right) \pm \frac{\Delta f_{352}}{f_{352} \ln (10)}\right)}{\log (666 \mathrm{GHz})-\log (352 \mathrm{GHz})},
$$

where $\Delta f_{\nu}$ represents the uncertainty in the fluxes, of $\alpha=0.11 \pm 0.49$ over these wavelengths. While this value is consistent with nonthermal emission, ${ }^{15}$ the spectral index itself is not sufficient to discriminate the emission mechanism.

The brightness temperature, $T_{b}$ can be approximated by

$$
T_{b} \sim\left(\frac{1}{2 k_{\mathrm{B}}}\right) \times \lambda^{2} \Delta S \times\left(\frac{D}{c \Delta t}\right)^{2}
$$

where $k_{\mathrm{B}}$ is Boltzmann's constant, $\Delta S$ is the change in flux over time $\Delta t, \lambda$ is the wavelength, $D$ is the distance to the source (389 pc), and $c$ is the speed of light. The change in $850 \mu \mathrm{m}$ flux $(\Delta S)$ and its corresponding time frame $(\Delta t)$ are derived from the light curve presented in Figure 5 with values of $373 \mathrm{mJy} \quad\left(\mathrm{mJy}=\mathrm{mJy}\right.$ beam $^{-1}$, for point sources) and $1661 \mathrm{~s}$, respectively. This results in a brightness temperature of $T_{b} \sim 6 \times 10^{4} \mathrm{~K}$ and a light-crossing distance of $3.3 \mathrm{au}$. Constraining the angular scale to a stellar radius of $R_{\star}=2.5 R_{\odot}$ (the average radius of the two components in the JW 566 binary system; Daemgen et al. 2012) rather than $c \Delta t$, results in an estimate of the upper range of the brightness temperature of $T_{b} \sim 5 \times 10^{9} \mathrm{~K}$, though the origin of the flare could indeed be generated by a region smaller in scale than $R_{\star}=2.5 R_{\odot}$. With a projected separation of $335 \mathrm{au}$, it is very unlikely that an interbinary interaction is occurring among the

\footnotetext{
15 Assuming a blackbody thermal spectrum, the temperature would need to be 4.9 $\mathrm{K}$ to reproduce such a flat spectral index.
}

known components. These calculations strongly favor nonthermal emission. The most likely scenario is that the flare was caused by gyrosynchrotron/synchrotron radiation emitted by a reconnection event in the strong magnetic fields present in the corona of these young stars (see, e.g., Salter et al. 2010). This magnetic reconnection briefly energizes nonthermal particles, which appear as a flare. The detection of such an event at $850 \mu \mathrm{m}$ suggests a very-high-energy acceleration of electrons. Polarimetry data is required to separately constrain the contributions of gyrosynchrotron and synchrotron emission; such data, however, are not available for this event.

\section{Summary}

In this paper, we presented 450 and $850 \mu \mathrm{m}$ SCUBA-2 observations of a bright flare associated with the T Tauri binary system JW 566 (R.A., decl. = 5:35:17.94,-5:16:11, J2000) obtained by the JCMT Transient Survey on 2016 November 26 (UT). The flare is measured to have a flux of $466 \pm$ $47 \mathrm{mJy}$ beam $^{-1}$ at $850 \mu \mathrm{m}$ and $500 \pm 107 \mathrm{mJy} \mathrm{beam}^{-1}$ at $450 \mu \mathrm{m}$ averaged over the observation (see Sections 4.1 and 4.2). We subdivided the 31 minute integration into nine intervals based on when the telescope scanned over the source and found a monotonic decrease of $337 \mathrm{mJy}^{\text {beam }}{ }^{-1}$ over $1661 \mathrm{~s}$ (see Section 4.3). Constraining the size scale of the flare origin to the light-crossing time of our observation and to the stellar radius of one of the binary components results in a range of brightness temperatures between $T_{b} \sim 6 \times 10^{4} \mathrm{~K}$ and $T_{b} \sim 5 \times 10^{9} \mathrm{~K}$ (see Section 5.2). The flat spectral index, the short variability timescale, and a large $T_{b}$ strongly indicate that the flare is a result of gyrosynchrotron/synchrotron emission, likely caused by a magnetic reconnection event.

The true timescale of the flare remains unknown, as there are no data available at other wavelengths during the time of our observation. The JCMT Transient Survey will continue through 2020 January, increasing the number of observations of the OMC $2 / 3$ field by a factor of $\sim 1.7$. Therefore, it is plausible that another burst of a similar magnitude will be detected. With new variable source detection methods (B. Lalchand et al. 2018 , in preparation), we will be able to identify future events within $\sim 24 \mathrm{hr}$ of the data being taken by the telescope in order to perform follow-up observations.

The authors wish to extend their gratitude to Dr. David Berry for useful discussions regarding the $450 \mu \mathrm{m}$ data reduction, to Dr. Helen Kirk for assistance with the JCMT GBS DR3 observations, to Dr. Jenny Hatchell who provided comments that strengthened this work, and to the anonymous referee for insightful comments.

D.J. is supported by NRC Canada and an NSERC Discovery Grant. G.J.H. is supported by general grants 11473005 and 11773002 awarded by the National Science Foundation of China. J.E.L. is supported by the Basic Science Research Program through the National Research Foundation of Korea (grant No. NRF-2018R1A2B6003423) and the Korea Astronomy and Space Science Institute under the R\&D program supervised by the Ministry of Science, ICT and Future Planning. A.H. is thankful for the support of the Netherlands Organisation for Scientific Research (NWO) under the VENI project 639.041.644. This paper makes use of the following ALMA data: ADS/JAO.ALMA\#2015.1.00669.S. 
The authors wish to recognize and acknowledge the very significant cultural role and reverence that the summit of Maunakea has always had within the indigenous Hawaiian community. We are most fortunate to have the opportunity to conduct observations from this mountain. The James Clerk Maxwell Telescope is operated by the East Asian Observatory on behalf of The National Astronomical Observatory of Japan; Academia Sinica Institute of Astronomy and Astrophysics; the Korea Astronomy and Space Science Institute; the Operation, Maintenance and Upgrading Fund for Astronomical Telescopes and Facility Instruments, budgeted from the Ministry of Finance (MOF) of China and administrated by the Chinese Academy of Sciences (CAS), as well as the National Key R\&D Program of China (No. 2017YFA0402700). Additional funding support is provided by the Science and Technology Facilities Council of the United Kingdom and participating universities in the United Kingdom and Canada. Additional funds for the construction of SCUBA-2 were provided by the Canada Foundation for Innovation. This research used the facilities of the Canadian Astronomy Data Centre operated by the National Research Council of Canada with the support of the Canadian Space Agency. This research has made use of the SIMBAD database, operated at CDS, Strasbourg, France (Wenger et al. 2000).

Facilities: JCMT, ALMA.

Software: astropy (Astropy Collaboration et al. 2013), matplotlib (Hunter 2007), aplpy (Robitaille \& Bressert 2012) starlink (Currie et al. 2014),

\section{ORCID iDs}

Steve Mairs (ib https://orcid.org/0000-0002-6956-0730 Bhavana Lalchand (1) https://orcid.org/0000-0003-1618-2921 Geoffrey C. Bower (1D https://orcid.org/0000-0003-4056-9982 Jan Forbrich (iD https://orcid.org/0000-0001-8694-4966 Graham S. Bell (i) https://orcid.org/0000-0003-0438-8228 Gregory J. Herczeg (iD https://orcid.org/0000-0002-7154-6065 Doug Johnstone (i) https://orcid.org/0000-0002-6773-459X Wen-Ping Chen (ib https://orcid.org/0000-0003-0262-272X Jeong-Eun Lee (iD https://orcid.org/0000-0003-3119-2087 Alvaro Hacar (iD https://orcid.org/0000-0001-5397-6961

\section{References}

Ali, B., \& Depoy, D. L. 1995, AJ, 109, 709

Anglada, G., Amado, P. J., Ortiz, J. L., et al. 2017, ApJL, 850, L6 Ansdell, M., Williams, J. P., van der Marel, N., et al. 2016, ApJ, 828, 46 Astropy Collaboration, Robitaille, T. P., Tollerud, E. J., et al. 2013, A\&A, 558, A33

Benz, A. O., \& Güdel, M. 2010, ARA\&A, 48, 241

Berry, D. S. 2015, A\&C, 10, 22

Billot, N., Morales-Calderón, M., Stauffer, J. R., Megeath, S. T., \& Whitney, B. 2012, ApJL, 753, L35

Bower, G. C., Plambeck, R. L., Bolatto, A., et al. 2003, ApJ, 598, 1140 Buckle, J. V., Hills, R. E., Smith, H., et al. 2009, MNRAS, 399, 1026 Chapin, E. L., Berry, D. S., Gibb, A. G., et al. 2013, MNRAS, 430, 2545 Cleeves, L. I., Bergin, E. A., Öberg, K. I., et al. 2017, ApJL, 843, L3 Coudé, S., Bastien, P., Kirk, H., et al. 2016, MNRAS, 457, 2139
Currie, M. J., Berry, D. S., Jenness, T., et al. 2014, in ASP Conf. Ser. 485, Astronomical Data Analysis Software and Systems XXIII, ed. N. Manset \& P. Forshay (San Francisco, CA: ASP), 391

Daemgen, S., Correia, S., \& Petr-Gotzens, M. G. 2012, A\&A, 540, A46

Dempsey, J. T., Friberg, P., Jenness, T., et al. 2013, MNRAS, 430, 2534

Drabek, E., Hatchell, J., Friberg, P., et al. 2012, MNRAS, 426, 23

Feigelson, E. D., Garmire, G. P., \& Pravdo, S. H. 2002, ApJ, 572, 335

Feigelson, E. D., \& Montmerle, T. 1999, ARA\&A, 37, 363

Forbrich, J., Menten, K. M., \& Reid, M. J. 2008, A\&A, 477, 267

Forbrich, J., Reid, M. J., Menten, K. M., et al. 2017, ApJ, 844, 109

Forbrich, J., Rivilla, V. M., Menten, K. M., et al. 2016, ApJ, 822, 93

Fraschetti, F., Drake, J. J., Cohen, O., \& Garraffo, C. 2018, ApJ, 853, 112

Furuya, R. S., Shinnaga, H., Nakanishi, K., Momose, M., \& Saito, M. 2003, PASJ, 55, L83

Gagne, M., Caillault, J.-P., \& Stauffer, J. R. 1995, ApJ, 445, 280

Gaia Collaboration, Brown, A. G. A., Vallenari, A., et al. 2018, arXiv:1804.09365

Garmire, G., Feigelson, E. D., Broos, P., et al. 2000, AJ, 120, 1426

Getman, K. V., Feigelson, E. D., Broos, P. S., Micela, G., \& Garmire, G. P. 2008a, ApJ, 688, 418

Getman, K. V., Feigelson, E. D., Grosso, N., et al. 2005, ApJS, 160, 353

Getman, K. V., Feigelson, E. D., Micela, G., et al. 2008b, ApJ, 688, 437

Gibb, A. G., Jenness, T., \& Economou, F. 2013, PICARD: A PIpeline for Combining and Analyzing Reduced Data, Starlink User Note 265 (Hilo, HI: Joint Astronomy Centre)

Glassgold, A. E., Najita, J., \& Igea, J. 2004, ApJ, 615, 972

Güdel, M. 2002, ARA\&A, 40, 217

Güdel, M., \& Benz, A. O. 1993, ApJL, 405, L63

Güdel, M., Dvorak, R., Erkaev, N., et al. 2014, Protostars and Planets VI (Tucson, AZ: University of Arizona Press)

Hacar, A., Tafalla, M., Forbrich, J., et al. 2018, A\&A, 610, A77

Herczeg, G. J., Johnstone, D., Mairs, S., et al. 2017, ApJ, 849, 43

Holland, W. S., Bintley, D., Chapin, E. L., et al. 2013, MNRAS, 430, 2513

Hunter, J. D. 2007, CSE, 9, 90

Jenness, T., Chapin, E. L., Berry, D. S., et al. 2013, SMURF: SubMillimeter User Reduction Facility, Astrophysics Source Code Library, ascl:1310.007 Johnstone, D., Hendricks, B., Herczeg, G. J., \& Bruderer, S. 2013, ApJ, 765, 133 Johnstone, D., Herczeg, G. J., Mairs, S., et al. 2018, ApJ, 854, 31 Jones, B. F., \& Walker, M. F. 1988, AJ, 95, 1755

Kackley, R., Scott, D., Chapin, E., \& Friberg, P. 2010, Proc. SPIE, 7740, 1 Kirk, H., Hatchell, J., Johnstone, D., et al. 2018, arXiv:1808.07952 Kounkel, M., Covey, K., Suárez, G., et al. 2018, AJ, 156, 84 Kounkel, M., Hartmann, L., Loinard, L., et al. 2014, ApJ, 790, 49 Kounkel, M., Hartmann, L., Loinard, L., et al. 2017, ApJ, 834, 142 Lammer, H., Zerkle, A. L., Gebauer, S., et al. 2018, A\&ARv, 26, 2 MacGregor, M. A., Weinberger, A. J., Wilner, D. J., Kowalski, A. F., \& Cranmer, S. R. 2018, ApJL, 855, L2

Mairs, S., Johnstone, D., Kirk, H., et al. 2015, MNRAS, 454, 2557

Mairs, S., Johnstone, D., Kirk, H., et al. 2017a, ApJ, 849, 107

Mairs, S., Lane, J., Johnstone, D., et al. 2017b, ApJ, 843, 55

Massi, M., Forbrich, J., Menten, K. M., et al. 2006, A\&A, 453, 959

Megeath, S. T., Gutermuth, R., Muzerolle, J., et al. 2012, AJ, 144, 192

Morales-Calderón, M., Stauffer, J. R., Hillenbrand, L. A., et al. 2011, ApJ, 733,50

Parsons, H., Dempsey, J. T., Thomas, H. S., et al. 2018, ApJS, 234, 22

Pascucci, I., Testi, L., Herczeg, G. J., et al. 2016, ApJ, 831, 125

Rab, C., Güdel, M., Padovani, M., et al. 2017, A\&A, 603, A96

Ricci, L., Testi, L., Natta, A., et al. 2010, A\&A, 512, A15

Robitaille, T., \& Bressert, E. 2012, APLpy: Astronomical Plotting Library in Python, Astrophysics Source Code Library, ascl:1208.017

Salter, D. M., Kóspál, Á, Getman, K. V., et al. 2010, A\&A, 521, A32 Salter, D. M., Hogerheijde, M. R., \& Blake, G. A. 2008, A\&A, 492, L21

Shu, F. H., Shang, H., Glassgold, A. E., \& Lee, T. 1997, Sci, 277, 1475 Skrutskie, M. F., Cutri, R. M., Stiening, R., et al. 2006, AJ, 131, 1163 Sossi, P. A., Moynier, F., Chaussidon, M., et al. 2017, NatAs, 1, 0055 Tsujimoto, M., Koyama, K., Kobayashi, N., et al. 2003, AJ, 125, 1537 Ward-Thompson, D., Di Francesco, J., Hatchell, J., et al. 2007, PASP, 119, 855 Wenger, M., Ochsenbein, F., Egret, D., et al. 2000, A\&AS, 143, 9 Yoo, H., Lee, J.-E., Mairs, S., et al. 2017, ApJ, 849, 69 